\title{
Evaluating the impact of financial toxicity among cancer clinical trial participants
}

\author{
Hala T. Borno, MD', Sylvia Zhang, MAS', Tracy Kuo Lin, $\mathrm{PhD}^{3}$, Li Zhang, PhD ${ }^{4}$, Nynikka R. Palmer, MPH, DrPH ${ }^{5}$, \\ Christopher Nguyen ${ }^{2}$, Celia Kaplan, MA, DrPH ${ }^{6}$, Charles J. Ryan, MD $^{7}$ \\ 1 Division of Hematology/Oncology, Department of Medicine, University of California, San Francisco \& Helen Diller Family Comprehensive Cancer \\ Center, San Francisco, California, ${ }^{2}$ Helen Diller Family Comprehensive Cancer Center, San Francisco, California, ${ }^{3}$ Department of Social and Behavioral \\ Sciences, Helen Diller Family Comprehensive Cancer Center, San Francisco, California \& Institute for Health \& Aging, University of California, San \\ Francisco, 4 Division of Hematology/Oncology, Department of Medicine \& Department of Epidemiology and Biostatistics, University of California, San \\ Francisco \& Helen Diller Family Comprehensive Cancer Center, San Francisco, California, 5 Division of General Internal Medicine \& Department of \\ Urology, Helen Diller Family Comprehensive Cancer Center, San Francisco, California, Zuckerberg San Francisco General Hospital, University of \\ California, San Francisco \& University of California, San Francisco, 6 Division of General Internal Medicine, Helen Diller Family Comprehensive Cancer \\ Center, San Francisco, California \& University of California, San Francisco, ${ }^{7}$ Division of Hematology, Oncology and Transplantation, University of \\ Minnesota, Minneapolis, Minnesota
}

Keywords: financial toxicity, clinical trial disparities, cancer

https://doi.org/10.53876/001c.30796

\section{International Journal of Cancer Care and Delivery}

Vol. 2, Issue 1, 2022

\section{INTRODUCTION}

Cancer precision medicine as part of standard of care (SOC) or clinical trials (CT) requires access to high-cost advanced diagnostics and therapeutics. ${ }^{1}$ The difference in out-ofpocket (OOP) costs associated with cancer treatment as part of SOC versus CTs remains limited. This study sought to evaluate the varying financial burden and toxicity among cancer patients receiving treatment at an academic medical center as part of a CT versus SOC.

\section{METHODS}

Patients presenting to medical oncology clinic at an academic medical center for a new or follow-up visit, who were English-speaking and able to provide informed consent, were approached for a one-time survey from July 2018 to August 2020. Study participants provided demographic, clinical, financial, and patient reported outcomes data. ${ }^{2} \mathrm{Fi}-$ nancial toxicity was measured using the Comprehensive Score for financial Toxicity (COST) patient reported outcome measure (supplement Table 1). ${ }^{3}$ The data was summarized using descriptive statistics, with t-tests and Chisquared tests were performed to identify differences between SOC versus CT participants for continuous and categorical variables, respectively.

\section{RESULTS}

As shown in Table 1, a total of 67 participants completed the survey. In the study, CT participants were significantly younger than SOC participants (CT, mean 57 years, $\mathrm{SD}=13.5$; SOC, mean $67, \mathrm{SD}=10.7$; $\mathrm{p}=0.002$ ). The mean time since diagnosis was significantly longer among SOC participants at 7.9 years $(\mathrm{SD}=7.3)$ compared to $\mathrm{CT}$ participants at 2.7 years $(S \mathrm{~S}=3.9)(\mathrm{p}=0.001)$. All study participants had solid tumors, except for $4 \mathrm{CT}$ participants with hematologic malignancies. The majority of participants had metastatic disease (SOC, 78\%; CT, 74\%). Most participants were nonHispanic white (SOC, 78\%; CT 71\%). The household income was significantly higher among SOC participants compared to CT participants, with a larger proportion reporting an income of $\$ 100,000$ or more (SOC, $52 \%$; CT, $19 \%$; $p<0.001$ ). A larger proportion of SOC participants were retired (56\%) compared to CT (26\%) participants ( $\mathrm{p}=0.01)$. A significantly higher proportion of CT participants reported OOP cost exceeding $\$ 1,000$ in the prior 30 days compared to SOC participants (SOC, 8.3\%; CT, 16.1\%, $\mathrm{p}<0.001$ ). The estimated monthly OOP cost was $\$ 742$ ( $\mathrm{SD}=\$ 1214)$ among CT participants compared to $\$ 278$ ( $S D=\$ 370$ ) among SOC participants $(p=0.05)$. Significantly more SOC participants felt very comfortable discussing cost with their cancer doctor compared to CT participants (SOC, 69\%; CT, 35\%; $\mathrm{p}=0.02$ ). As shown in Supplement Table 1, a higher level of financial toxicity was observed among CT participants (COST PROM score $=16.86, \mathrm{SD}=10.31)$ compared to SOC participants $($ COST PROM score $=19.77, \mathrm{SD}=4.91)(\mathrm{p}=0.03)$. As shown in Figure 1, the participants insured with Medicare/ Medicaid reported similar level of financial toxicity whether they were offered SOC versus enrolled in a CT. However, participants with private insurance enrolled in CTs reported a lower COST score, as compared to SOC participants, indicating higher financial toxicity; due to the small sample, this pattern is purely descriptive.

\section{DISCUSSION}

This study observed significantly higher OOP cost and financial toxicity among participants receiving treatment within a CT compared to SOC. These differences in self-reported OOP cost and financial toxicity may be attributed to increased frequency of CT appointments, longer duration of clinic visits, additional CT required labs and scans, or greater distances traveled to CT site. ${ }^{4-7}$ Coupled with variable increases in cost-sharing by insurance type, these 
Table 1. Participant Characteristics

\begin{tabular}{|c|c|c|c|c|c|}
\hline \multirow[b]{2}{*}{ Characteristic } & \multicolumn{2}{|c|}{ Standard of Care $(\mathrm{N}=36)$} & \multicolumn{2}{|c|}{ Clinical Trial $(\mathrm{N}=31)$} & \multirow[b]{2}{*}{$p$-value } \\
\hline & $n$ & $\%$ & $n$ & $\%$ & \\
\hline Age, mean (SD), years & $67(10.7)$ & & $57(13.5)$ & & $0.002^{* *}$ \\
\hline Race/Ethnicity & & & & & 0.34 \\
\hline White & 28 & 77.8 & 22 & 71.0 & \\
\hline Black/African American & 1 & 2.8 & 0 & 0.0 & \\
\hline Asian & 2 & 5.6 & 2 & 6.5 & \\
\hline Native Hawaiian/Other Pacific Islander & 1 & 2.8 & 1 & 3.2 & \\
\hline Hispanic or Latino & 3 & 8.3 & 5 & 16.1 & \\
\hline American Indian or Alaska Native & 0 & 0.0 & 1 & 3.2 & \\
\hline Unknown & 1 & 2.8 & 0 & 0.0 & \\
\hline Household Income & & & & & $0.01^{* *}$ \\
\hline$\$ 24,999$ or less & 2 & 5.6 & 3 & 9.7 & \\
\hline$\$ 25,000$ to $\$ 55,999$ & 8 & 22.2 & 10 & 32.3 & \\
\hline$\$ 56,000$ to $\$ 99,999$ & 6 & 16.7 & 12 & 38.7 & \\
\hline$\$ 100,000$ or more & 19 & 52.8 & 6 & 19.4 & \\
\hline Unknown & 1 & 2.8 & 0 & 0.0 & \\
\hline Highest Education Attained & & & & & 0.40 \\
\hline High school degree or less & 7 & 19.5 & 10 & 32.3 & \\
\hline College degree or equivalent & 15 & 41.7 & 14 & 45.2 & \\
\hline Graduate degree or equivalent & 14 & 38.9 & 7 & 22.6 & \\
\hline Marital Status & & & & & 0.63 \\
\hline Never married & 2 & 5.6 & 2 & 5.6 & \\
\hline Married or civil partnership & 29 & 80.6 & 20 & 64.5 & \\
\hline Divorced, separated, or widowed & 4 & 11.1 & 6 & 19.4 & \\
\hline Living with partner & 1 & 2.8 & 2 & 6.5 & \\
\hline Unknown & 0 & 0.0 & 1 & 3.2 & \\
\hline Employment Status & & & & & $0.02^{*}$ \\
\hline Full-time & 10 & 27.8 & 6 & 19.4 & \\
\hline On disability & 1 & 2.8 & 9 & 29.0 & \\
\hline Unemployed & 5 & 13.9 & 4 & 12.9 & \\
\hline Retired & 20 & 55.6 & 8 & 25.8 & \\
\hline Other/Unknown & 0 & 0.0 & 4 & 12.9 & \\
\hline Insurance Type & & & & & $0.01^{* *}$ \\
\hline Private & 13 & 36.1 & 16 & 41.9 & \\
\hline Medicaid/Medicare & 21 & 58.3 & 9 & 29.0 & \\
\hline Veterans Affairs/Military & 1 & 2.8 & 1 & 3.2 & \\
\hline Other & 1 & 2.8 & 5 & 16.1 & \\
\hline \multicolumn{6}{|l|}{ Cancer Type } \\
\hline Hematologic Malignancy & 0 & 0 & 4 & 12.9 & $0.03^{*}$ \\
\hline Solid Tumor & 36 & 100.0 & 27 & 87.1 & \\
\hline Years Since Diagnosis (Mean, SD) & $7.97(7.3)$ & & $2.68(3.9)$ & & $0.001^{* *}$ \\
\hline Mode(s) of travel to last appointment & & & & & 0.22 \\
\hline Private Vehicle & 29 & 82.9 & 24 & 77.4 & \\
\hline Public Transportation (ex. bus, train, shuttle) & 3 & 8.6 & 7 & 22.6 & \\
\hline Taxi & 4 & 11.4 & 2 & 6.5 & \\
\hline Walk & 1 & 2.9 & 1 & 3.2 & \\
\hline Airplane & 3 & 8.6 & 1 & 3.2 & \\
\hline
\end{tabular}




\begin{tabular}{|c|c|c|c|c|c|}
\hline \multirow[b]{2}{*}{ Characteristic } & \multicolumn{2}{|c|}{ Standard of Care $(\mathrm{N}=36)$} & \multicolumn{2}{|c|}{ Clinical Trial $(\mathrm{N}=31)$} & \multirow[b]{2}{*}{$p$-value } \\
\hline & $n$ & $\%$ & $n$ & $\%$ & \\
\hline Other (Uber, Lyft, etc.) & 0 & 0.0 & 3 & 9.7 & \\
\hline $\begin{array}{l}\text { How many hours out of your day did your last } \\
\text { appointment for cancer care/treatment take (including } \\
\text { travel time)? }\end{array}$ & & & & & 0.06 \\
\hline Less than 1 hour & 1 & 2.8 & 1 & 3.2 & \\
\hline 1 to 3 hours & 11 & 30.6 & 2 & 6.5 & \\
\hline 3 to 5 hours & 10 & 2.8 & 6 & 19.4 & \\
\hline 5 to 8 hours & 9 & 25.0 & 12 & 38.7 & \\
\hline More than 8 hours & 5 & 13.9 & 10 & 32.3 & \\
\hline $\begin{array}{l}\text { About how much out of pocket costs did you have } \\
\text { related to cancer care/treatment in the last month? } \\
\text { This includes hotel/lodging costs, tests, medications, } \\
\text { copays, etc. }\end{array}$ & & & & & $<0.001^{* * *}$ \\
\hline Less than $\$ 50$ & 10 & 27.8 & 1 & 3.2 & \\
\hline$\$ 50$ to $\$ 100$ & 11 & 30.6 & 2 & 6.5 & \\
\hline$\$ 100$ to $\$ 300$ & 3 & 8.3 & 9 & 29.0 & \\
\hline$\$ 300$ to $\$ 500$ & 0 & 0.0 & 7 & 22.6 & \\
\hline$\$ 500$ to $\$ 700$ & 8 & 22.2 & 5 & 16.1 & \\
\hline$\$ 700$ to $\$ 1000$ & 1 & 2.8 & 1 & 3.2 & \\
\hline More than $\$ 1000$ & 3 & 8.3 & 5 & 16.1 & \\
\hline Unknown & 0 & 0.0 & 1 & 3.2 & \\
\hline $\begin{array}{l}\text { Estimated monthly out of pocket costs, mean (SD), } \\
\text { dollars }\end{array}$ & $\$ 278(\$ 370)$ & & $\$ 742(\$ 1214)$ & & $0.05^{*}$ \\
\hline Do you usually need to arrange overnight lodging? & & & & & 0.08 \\
\hline Yes & 9 & 25.0 & 14 & 45.2 & \\
\hline No & 27 & 75.0 & 17 & 54.8 & \\
\hline $\begin{array}{l}\text { How comfortable do you feel bringing up healthcare } \\
\text { cost related issues with your cancer doctor? }\end{array}$ & & & & & $0.02^{*}$ \\
\hline Uncomfortable & 4 & 11.1 & 11 & 35.5 & \\
\hline Somewhat comfortable & 7 & 19.4 & 8 & 25.8 & \\
\hline Very comfortable & 25 & 69.4 & 11 & 35.5 & \\
\hline Unknown & 0 & 0.0 & 1 & 3.2 & \\
\hline
\end{tabular}

$* \mathrm{p}<0.05$

*** $\mathrm{p}<0.01$

$* * * * 0.001$

may contribute to the growing financial burden on patients enrolled in CTs. Despite this observation, CT participants in this study were less likely to feel comfortable bringing up healthcare cost related issues with their cancer doctor. While this study is limited by a small sample size, large and meaningful differences were identified between the two groups, suggesting that there is a critical need to further examine the added financial toxicity placed on patients receiving cancer treatment in the context of a CT. Given the racial/ethnic disparities observed in oncology CTs, ${ }^{8}$ the financial burden associated with participation must be closely examined and addressed to promote equity in access to CTs.

Submitted: November 09, 2021 PST, Accepted: December 13,

2021 PST 


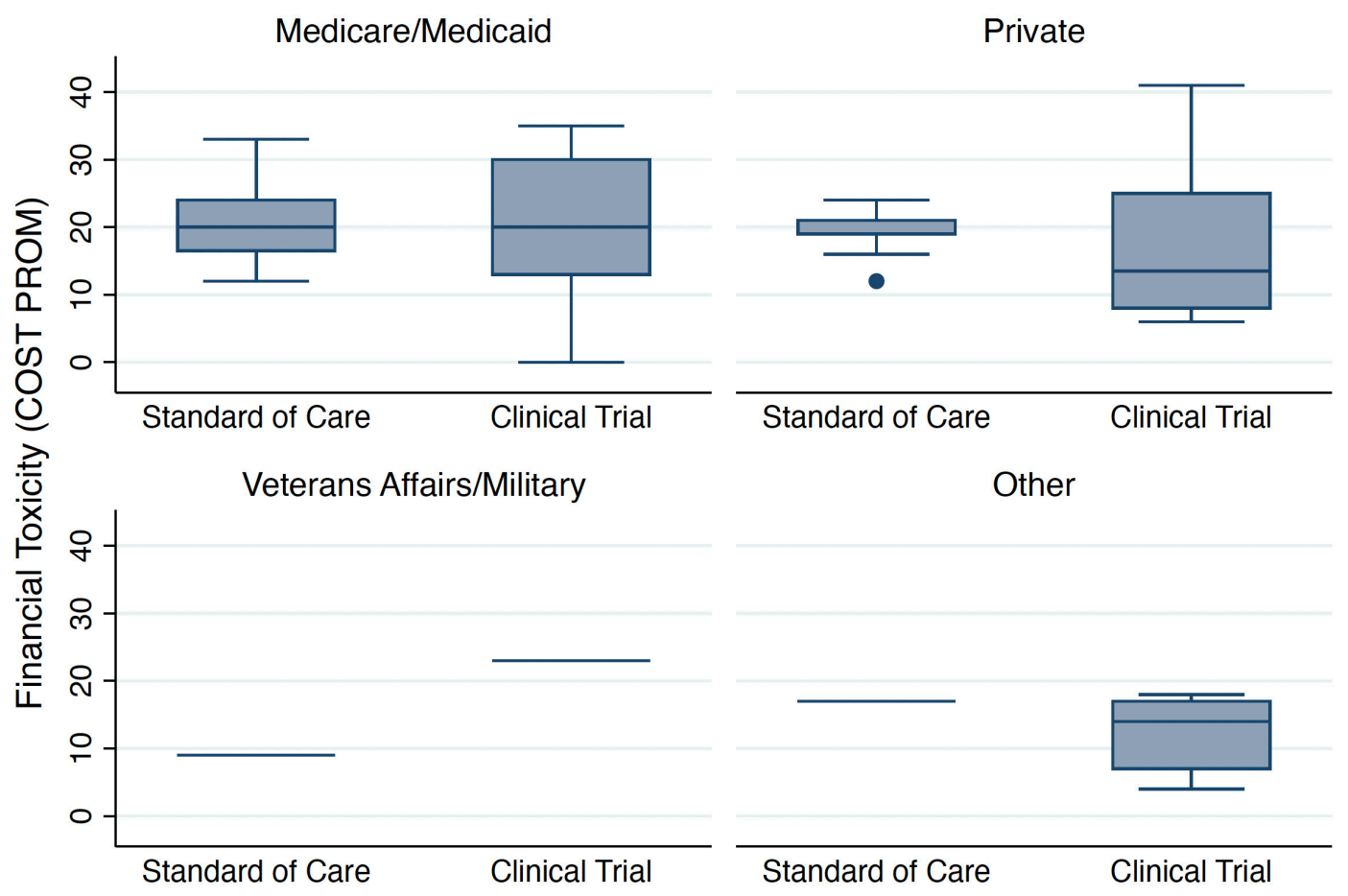

Figure 1. Boxplots of Financial Toxicity (COST PROM) for Standard of Care versus Clinical Trial participants stratified by insurance type

This is an open-access article distributed under the terms of the Creative Commons Attribution 4.0 International License (CCBY-SA-4.0). View this license's legal deed at https://creativecommons.org/licenses/by-sa/4.0 and legal code at https://creativecommons.org/licenses/by-sa/4.0/legalcode for more information. 


\section{REFERENCES}

1. Conti R, Veenstra DL, Armstrong K, Lesko LJ, Grosse SD. Personalized medicine and genomics: challenges and opportunities in assessing effectiveness, cost-effectiveness, and future research priorities. Med Decis Making. 2010;30(3):328-340. do i:10.1177/0272989X09347014

2. de Souza JA, Yap BJ, Wroblewski K, et al. Measuring financial toxicity as a clinically relevant patient-reported outcome: The validation of the COmprehensive Score for financial Toxicity (COST). Cancer. 2017;123(3):476-484. doi:10.1002/cncr.30369

3. de Souza JA, Yap BJ, Hlubocky FJ, et al. The development of a financial toxicity patient-reported outcome in cancer: The COST measure. Cancer. 2014;120(20):3245-3253. doi:10.1002/cncr.28814

4. Huey RW, George GC, Phillips P, et al. PatientReported Out-of-Pocket Costs and Financial Toxicity During Early-Phase Oncology Clinical Trials. Oncologist. 2021;26(7):588-596. doi:10.1002/onco.137 $\underline{67}$
5. Chino F, Zafar SY. Financial Toxicity and Equitable Access to Clinical Trials. Am Soc Clin Oncol Educ Book. 2019;39:11-18. doi:10.1200/EDBK 100019

6. Nipp RD, Lee H, Powell E, et al. Financial Burden of Cancer Clinical Trial Participation and the Impact of a Cancer Care Equity Program. Oncologist. 2016;21(4):467-474. doi:10.1634/theoncologist.201 $\underline{5-0481}$

7. Borno HSA, Chang E, Zhang L, Charles R. At what cost to clinical trial enrollment? A retrospective study of patient travel burden in cancer clinical trials. Oncologist. Published online 2018.

8. Loree JM, Anand S, Dasari A, et al. Disparity of Race Reporting and Representation in Clinical Trials Leading to Cancer Drug Approvals From 2008 to 2018. JAMA Oncology. 2019;5(10):e191870. doi:10.100 $1 /$ jamaoncol.2019.1870 
Supplement Table 1. COmprehensive Score for financial Toxicity (COST) Patient-Reported Outcome Measures

\begin{tabular}{|c|c|c|c|c|}
\hline & & $\begin{array}{l}\text { Standard of } \\
\text { Care } \\
(\mathrm{N}=36)\end{array}$ & $\begin{array}{l}\text { Clinical } \\
\text { Trial } \\
(\mathrm{N}=31)\end{array}$ & \\
\hline & Survey Question, Range 0-4a, Mean (SD) & & & $p$-value \\
\hline 1 & $\begin{array}{l}\text { I know that I have enough money in savings, retirement, or assets to cover } \\
\text { the costs of my treatment. }\end{array}$ & $2.20(1.51)$ & $\begin{array}{l}0.94 \\
(1.18)\end{array}$ & $0.01^{* *}$ \\
\hline 2 & My out-of-pocket medical expenses are more than I thought they would be. & $1.19(1.17)$ & $\begin{array}{l}1.83 \\
(1.37)\end{array}$ & 0.31 \\
\hline 3 & $\begin{array}{l}\text { I worry about the financial problems I will have in the future as a result of my } \\
\text { illness or treatment }\end{array}$ & $1.56(1.34)$ & $\begin{array}{l}1.35 \\
(1.31)\end{array}$ & 0.37 \\
\hline 4 & I feel I have no choice about the amount of money I spend on care. & $2.17(1.54)$ & $\begin{array}{l}1.06 \\
(1.31)\end{array}$ & $0.04^{*}$ \\
\hline 5 & I am frustrated that I cannot work or contribute as much as I usually do. & $1.14(1.38)$ & $\begin{array}{l}1.45 \\
(1.52)\end{array}$ & 0.61 \\
\hline 6 & I am satisfied with my current financial situation. & $2.47(1.44)$ & $\begin{array}{l}1.32 \\
(1.30)\end{array}$ & $0.03^{*}$ \\
\hline 7 & I am able to meet my monthly expenses. & $3.25(1.08)$ & $\begin{array}{l}2.03 \\
(1.30)\end{array}$ & $0.003^{* *}$ \\
\hline 8 & I feel financially stressed. & $1.17(1.13)$ & $\begin{array}{l}1.87 \\
(1.23)\end{array}$ & $0.02^{*}$ \\
\hline 9 & I am concerned about keeping my job and income, including work at home. & $0.81(1.28)$ & $\begin{array}{l}2.26 \\
(1.59)\end{array}$ & $0.002^{* *}$ \\
\hline 10 & $\begin{array}{l}\text { My cancer or treatment has reduced my satisfaction with my present } \\
\text { financial situation. }\end{array}$ & $1.19(1.28)$ & $\begin{array}{l}1.30 \\
(1.48)\end{array}$ & 0.65 \\
\hline \multirow[t]{2}{*}{11} & I feel in control of my financial situation. & $2.58(1.13)$ & $\begin{array}{l}1.42 \\
(1.23)\end{array}$ & $0.007^{* *}$ \\
\hline & Overall COST Score, mean (SD) ${ }^{b}$ & $\begin{array}{l}19.77 \\
(4.91)\end{array}$ & $\begin{array}{l}16.86 \\
(10.31)\end{array}$ & $0.03^{*}$ \\
\hline
\end{tabular}

aItems 2, 3, 4, 5, 8, 9, and 10 were reverse coded

bower COST scores indicate higher toxicity (0-44)

*p $<0.05$

*** $p<0.01$

$* * * 200.001$ 